\section{Allain's warnings unheeded}

SIR - I write to respond to your leading article (Nature 364, 267; 1993) on the case of Jean-Pierre Allain. The trial and the verdict do indeed raise important issues of principle, but they go much further than the issue of when "whistle-blowers should blow the whistle". More important, as the Royal College of Pathologists argued in its statement earlier this year, is the issue of whether "doctors who give their professional opinions in good faith ... . can be held legally responsible for the failure of that advice to be taken".

You appear to blame Allain for "not blowing the whistle between the beginning of 1984 and October 1985". The Lancet points out "Between knowledge and action there is always an interval because the knowledge itself may prove insecure and because it takes time to move from research base to action" $(342,188$; 1993). It is therefore not just a matter of detail that your starting date "beginning of 1984 " is almost exactly one year too early.

What did Allain (or anybody else) know early in 1985? In 1983-84, Allain coordinated a study of the immunological and virological status and type and origin of blood products in French multitransfused patients. The data were analysed by December 1984, first submitted for publication on 7.January 1985 , eventually accepted for publication by Blood in April 1985 and published in October 1985.

During that period, the conclusions of the study would have been known to the other 27 participants, who included the directors of major haemophilia and transfusion centres in France, distinguished immunologists and virologists, Professor Luc Montagnier of the Institut Pasteur among them. The report was sent to the director of the French National Blood Transfusion Centre (CNTS) and was the basis of Allain's written advice, in January 1985 , to the director and president of CNTS that heat treatment of blood products should begin without delay. But the paper in Blood also reveals the continuing uncertainties at that time. Thus the abstract of the paper concludes:

This study has shown the prominent role of LAV in the occurrence of immunological disturbances in multi-transfused patients. However, allogenic or altered proteins present in factor VIII but not in factor IX concentrates seem to play a role of immunocomprising agents. The interplay between LAV and additional factors possibly leading to acquired immuno-deficiency syndrome remains to be analysed.

Although Allain's advocacy of heat treatment had by then been supported elsewhere, doubts about the heat treatment were also prevalent; thus Bird et al. stated in January 1985 (Lancet $\mathbf{i}, 162-163$ ) that: there is therefore a considerable danger that the unproven benefits of heat treatment will be offset by potential risks, one of which — antibody-inhibitor formation - would be irreversible.

In February 1985 there was further preliminary but clear evidence that heat treated factor VIII did not cause seroconversion.

Thus the debate about the heat treatment of antihaemophilia preparations was already in the public domain in science; it is wrong therefore to draw, as you do, an analogy with "an employee of a defence research establishment who knows that some new weapon is unsafe".

On Allain's professional responsibility, as a practising physician he was responsible for a small group of haemophiliacs, none of whom seroconverted in 1985 . The knowledge that informed his own clinical practice had already been made available, as I have explained, to others with similar responsibility to patients.

Moreover, Allain had no responsibility for national strategy; he was in the third tier of administration in the CNTS, with responsibilities for a research section. His actions in alerting the administration are a matter of public record, as are those of his wife, Dr Helen Lee. For example, in April 1985 , she expressed their views forcefully to more than 200 of France's haematologists. The Royal College of Pathologists' working party on the Allain affair concluded:

. in the months following the writing of his letter and until the universal introduction of heated factor VIII was imminent, he and his wife did all that could be expected of them to persuade those in administrative charge of CNTS to follow their advice.

It is clear that in the early part of 1985 , there were still doubts in France, and that those responsible for national policy could draw attention to deficiencies in Allain's arguments. Some interpreted his advice as a panic reaction (which also happened elsewhere, in Canada for example).

For my part, I endorse the view of the Royal College of Pathologists that "it is wholly inimical to the pursuit of medical research and to the high standards of medical practice" that physicians can be prosecuted and convicted because their advice is not followed.

\section{Kelth Peters}

Office of the Regius Professor of Physic, School of Clinical Medicine,

Addenbrooke's Hospital, Cambridge CB2 2SP, UK

\section{Lorenzo's oil}

SIR - Fred S. Rosen's review of Lorenzo's Oil (Nature 361, 695; 1993) is typical of the backlash against the film in certain sections of the medical and scientific press. We are all, of course, entitled to our opinions. But Rosen's central reservations about the film are whether 'Lorenzo's oil' is effective in the treatment of adrenoleukodystrophy (ALD) and whether the film overstates its effectiveness. His comments in this respect demand a response.

Lorenzo's oil, the discovery of which is credited to Lorenzo's father, Augusto Odone, clearly reduces the levels of the offending long-chain fatty acids in the blood of patients with ALD. Such reduction is one of the main therapeutic goals for researchers working on the disease. What is not clear, as Rosen points out, is whether the oil prevents the onset of the disease in the target organs (the central nervous system and adrenal glands). But, given the pleiotropic effect of the ALD gene, with its variable phenotype and the disease's late onset in 25 per cent of patients, the only way in which the effectiveness of this therapy can be assessed is in a carefully controlled, long-term study (H. Moser J. NIH Res. 5, 35-36; 1993). In the meantime, the only hope for boys suffering from ALD is Lorenzo's oil or possibly bone marrow transplantation; even physicians who have publicly questioned the efficacy of the oil are continuing to prescribe it.

Further, Rosen is concerned that boys with ALD may develop thrombocytopaenia from taking the oil. However, W. $H$. Zinkham et al. have recently reported (New Engl. J. Med. 328, 1126; 1993) that there is no serious bleeding, although they do advise that platelet counts should be monitored. This would therefore seem to be a minor concern compared with the clinical course of ALD. Rosen thinks that the Odones have not set a good example for biomedical progress. I disagree. In devising the oil and setting up the Myelin Project to accelerate research into remyelination of the central nervous system, the Odones have shown that lay people can make important intellectual and supportive contributions to science.

At the very least, the film will help to educate the public about the medical aspects of a rare disease, raise funds for research and underline the need for animal research in developing treatments for incurable diseases. Perhaps the filmmakers should have shown a little more caution in stating the effectiveness of the oil. But regardless of whether the treatment works in the long run, the Odones' achievement should not be underestimated: they have set a magnificent example for medical progress, which few of us would be able to emulate.

lan D. Duncan

School of Veterinary Medicine,

Department of Medical Science,

University of Wisconsin,

Madison,

Wisconsin 53706-1102, USA 